\title{
Longitudinal changes on liver proton density fat fraction differ between liver segments
}

\author{
Jan Syväri ${ }^{1}$, Daniela Junker ${ }^{1}$, Lisa Patzelt ${ }^{1}$, Katharina Kappo ${ }^{2}$, Loubna Al Sadat ${ }^{2}$, Sonia Erfanian $^{2}$, \\ Marcus R. Makowski ${ }^{1}$, Hans Hauner ${ }^{2,3}$, Dimitrios C. Karampinos ${ }^{1}$ \\ ${ }^{1}$ Department of Diagnostic and Interventional Radiology, School of Medicine, Technical University of Munich, Munich, Germany; ${ }^{2}$ Institute for \\ Nutritional Medicine, Else Kroener-Fresenius-Center of Nutritional Medicine, School of Medicine, Technical University of Munich, Munich, \\ Germany; ${ }^{3}$ German Center for Diabetes Research, Helmholtz Diabetes Center, Neuherberg, Germany \\ Correspondence to: Jan Syväri. Department of Diagnostic and Interventional Radiology, Klinikum rechts der Isar, Technische Universität München, \\ Ismaninger Str. 22, 81675 Munich, Germany. Email: jan.syvaeri@tum.de.
}

Background: To study the spatial heterogeneity of liver fat fraction changes during a long-term lifestyle intervention study using magnetic resonance imaging (MRI).

Methods: Thirty-two subjects underwent two MRI-scans in a span of one year. A chemical shift encodingbased water-fat separation method was applied to measure liver proton density fat fraction (PDFF) maps. The PDFF changes in the two liver lobes and the Couinaud segments were compared with the mean liver PDFF change.

Results: The slope of the relationship between mean liver PDFF changes and PDFF liver lobe changes was higher in the right compared to the left lobe (slope mean PDFF whole liver $\sim$ mean PDFF right lobe $=1.08$, slope mean PDFF whole liver $\sim$ mean PDFF left lobe $=0.93, \mathrm{P}<0.001$ ). The highest slope of agreement between PDFF changes in each specific liver segment and mean liver PDFF changes was observed in segment VII (slope =1.12). The lowest slope of agreement between PDFF changes in each specific liver segment and mean liver PDFF changes was observed in segment I (slope $=0.77$ ).

Conclusions: Larger PDFF changes in the right liver lobe were observed compared to PDFF changes in the left liver lobe (LLL) in subjects with both increasing and decreasing mean liver PDFF after one year. The results are in line with the existing literature reporting a heterogeneous spatial distribution of liver fat and highlight the need to spatially resolve liver fat fraction changes in longitudinal studies.

Keywords: Lifestyle intervention study; liver fat fraction spatial heterogeneity; quantitative MRI; Dixon MRI; proton density fat fraction (PDFF)

Submitted Jul 15, 2020. Accepted for publication Nov 19, 2020.

doi: 10.21037/qims-20-873

View this article at: http://dx.doi.org/10.21037/qims-20-873

\section{Introduction}

A sedentary lifestyle and chronic over-nutrition are the leading causes for the increasing rates of overweight, metabolic syndrome and subsequently type 2 diabetes in many countries around the globe (1-3). The metabolic syndrome is associated with fat deposition in the liver, and eventually with the occurrence of nonalcoholic fatty liver disease (NAFLD) (4), which is a main cause of chronic liver disease (5). Specifically, patients with NAFLD are at high risk of developing liver fibrosis and liver cirrhosis leading to irreversible liver dysfunction and eventually are at high risk for liver carcinoma or other fatal complications (6). It is well known that early fat deposition in the liver caused by over-nutrition and the lack of exercise is reversible $(7,8)$. Therefore, tracking liver fat changes in patients with NAFLD is important for both risk assessment and treatment.

Measuring liver fat is feasible using either invasive or 
non-invasive methods. Liver biopsy is considered the gold standard for measuring liver fat in single locations (9). However, since liver biopsy is highly invasive, its use in longitudinal studies can be associated with strong discomfort for the patient and other complications. Singlevoxel magnetic resonance spectroscopy (MRS) has been shown to be a very reliable way of non-invasively measuring the liver proton density fat fraction (PDFF) (10). However, single-voxel MRS has limited spatial coverage as it typically reports the result on one specific area of the liver per measurement. Therefore, neither biopsy nor single-voxel MRS can cover the whole liver and therefore cannot detect any heterogeneity in liver fat deposition. In addition, repetitive measurements could become difficult to perform using techniques with limited spatial coverage, since the selection of the exact same region during the measurement at different timepoints is more difficult to execute.

Magnetic resonance imaging (MRI)-based PDFF mapping has been alternatively emerging as a reliable and accurate technique for spatially resolving liver fat fraction non-invasively. MRI-based PDFF has been validated as a biomarker in comparison with MRS and biopsies $(11,12)$. MRI-based liver PDFF mapping adds the advantage of spatially covering the whole organ with adequate resolution and therefore enables the investigation of liver fat distribution throughout the whole organ taking into consideration any differences between the individual Couinaud liver segments. In addition, as the whole liver is covered during the scan, placing of regions of interests (ROI) after the scan can be easily compared to previous measurements of the same subject, which is important for longitudinal measurements.

Multiple cross-sectional studies have reported heterogeneous liver fat distribution across different lobes and segments (13-16). Recently, Fazeli Dehkordy et al. (17) showed, based on PDFF mapping, a heterogeneity in liver fat distribution both at baseline and after bariatric weightloss surgery and reported right lobe segments having higher $\mathrm{PDFF}$ at baseline and a more rapid reduction in liver PDFF than left lobe segments. In this study, all subjects lost weight after surgery and showed a decreasing PDFF across all liver segments and lobes. However, it is not known how the spatial distribution of the PDFF is changing in a longitudinal setting, where subjects participating in a longterm lifestyle intervention study might either gain or lose weight during the intervention.

The purpose of our study was to study the spatial heterogeneity of PDFF changes in subjects participating in a long-term lifestyle intervention study and show variable changes in body weight.

\section{Methods}

\section{Subjects}

Thirty-two subjects (18 women and 14 men) participating in a German wide prospective, randomized, multicenter lifestyle intervention study at the Else Kröner-FreseniusCenter for Nutritional Medicine, Technical University of Munich, with MRI scans at two different time points within a time interval of one year were included in the present analysis. Inclusion criteria were age between 18 and 75 years and a high risk of developing diabetes mellitus type 2 by scoring more than 50 points in the screening questionnaire "German Diabetes Risk Test" (18). The subjects received nutritional counseling for one year covering multiple appointments. It included discussing nutritional protocols, information and recommendations on healthy eating and moderate energy restriction for losing weight, and individual guidance for more exercise. Further inclusion and exclusion criteria and more details about the intervention can be found at (19). The present dataset includes 17 subjects scanned before the beginning of the intervention and after 1 year and 15 subjects scanned after the end of the intervention at two time points with an interval of 1 year when weight regain was frequently occurring.

\section{MRI measurements}

The study was approved by an ethics committee and all subjects gave written informed consent before participation in the study including repeated MRI scanning. MRI was performed on a whole-body-MRI-scanner (Ingenia, 3.0T, Philips Healthcare, Best, The Netherlands) using the built in 12-channel posterior and 16-channel anterior coil.

The MRI acquisition consisted of an axial six-echo multiecho 3D spoiled gradient echo sequence for chemical shift-encoding based water-fat separation of the abdomen using bipolar gradient readouts. The acquisition time was $14.1 \mathrm{~s}$ and was performed with a single breath hold and covered the liver, with the following parameters: TR/TE1/ $\Delta \mathrm{TE}=7.8 / 1.3 / 1.1 \mathrm{~ms}$, echoes $=6, \mathrm{FOV}=300 \times 402.6 \times 150 \mathrm{~mm}^{3}$, acquisition voxel size $=1.97 \times 3.01 \times 6.00 \mathrm{~mm}^{3}$, acquisition matrix $=152 \times 133$, SENSE reduction factor $=2.2(\mathrm{RL}) \times 1.2$ $(\mathrm{FH})$, frequency direction $=\mathrm{A} / \mathrm{P}$, bandwidth $=1,522.8 \mathrm{~Hz}$, $\mathrm{NSA}=1$, flip angle $=3^{\circ}$. 
Complex multi-echo gradient-echo images were generated and provided as input to the fat quantification algorithm provided by the vendor (mDixon Quant, Philips Healthcare), including the following steps: a phase correction step was performed first to address eddy current-induced phase errors. Field inhomogeneityinduced misregistration effects and chemical shift-induced mis-registration effects were minimized by using the maximum receiver bandwidth. A complex-based waterfat decomposition was performed next using a single $T_{2}{ }^{*}$ correction and a pre-calibrated fat spectrum accounting for the multi-peak nature of the fat spectrum. A sevenpeak fat spectrum model was used (20). The PDFF map was computed as the ratio of the fat signal over the sum of fat and water signals and the magnitude discrimination approach was used to reduce noise bias effects (21).

\section{Segmentation of liver PDFF}

In each Couinaud segment (22) of the liver, a circular ROI with a diameter of $20 \mathrm{~mm}$ (volume of $\sim 1,885 \mathrm{~mm}^{3}$ ) was placed by a researcher (JS) with 2 years of experience in liver segmentation with 3D Slicer as described by Tang (23). Liver PDFF was calculated per ROI in each segment. Mean liver PDFF was calculated as the mean of all ROIs from each segment. For mean PDFF of left liver lobe (LLL), the mean PDFF of the ROIs of segments I, II, III, IVa and IVb, and for the right liver lobe (RLL) of the segments V, VI, VII and VIII, respectively, were calculated.

For inter-observer analysis, 7 subjects $(21.9 \%)$ were segmented by a second observer (LP, 1 year of experience in liver segmentation) and the inter-observer correlations for mean PDFF of whole liver, liver lobes and liver segments were calculated to study the reproducibility of the segmentation.

\section{Statistical analysis}

Statistical analyses were performed with RStudio [RStudio Team (2016). RStudio: Integrated Development for R. RStudio, Inc., Boston, MA, URL http://www.rstudio.com/]. Linear regression was calculated with Pearson correlation coefficient analysis with 0.05 level of significance. Twosided paired $t$-test was used to calculate differences in fat content between liver lobes with 0.05 level of significance. A statistical test was performed to compare the slope between PDFF changes in the LLL and mean PDFF changes and the slope between PDFF changes in the RLL and mean
PDFF changes, using a $\mathrm{z}$ statistic defined by the difference between the slopes of the regression lines divided by the standard error of the differences between the slopes (24). The intraclass correlation coefficient (ICC) was used to calculate inter-observer agreement.

\section{Results}

\section{Subjects' characteristics}

The age range of the scanned subjects was from 24 to 76 (mean: 54.3) years at the timepoint of the first MRI scan. Subjects showed weight changes ranging from $+11.1 \mathrm{~kg}$ $(+11.4 \%)$ to $-11.4 \mathrm{~kg}(-10.1 \%)$ with a mean absolute change of $3.8 \mathrm{~kg} ; 17$ subjects showed weight gain (mean weight increase: $+3.8 \pm 2.9 \mathrm{~kg}$ ) and 15 subjects showed weight loss over the period of 1 year (mean weight decrease: $-4.0 \pm 3.5 \mathrm{~kg}$ ). The subjects did not show different changes in weight depending on the starting point (starting point before intervention: $-1.2 \pm 4.9 \mathrm{~kg}$, starting point after intervention: $+1.4 \pm 5.0 \mathrm{~kg})$.

\section{Inter-observer analysis}

The ICC for PDFF values between the two observers per liver segment was 0.998 for segment I, 0.994 for segment II, 0.994 for segment III, 0.989 for segment IVa, 0.998 for segment IVb, 0.986 for segment V, 0.997 for segment VI, 0.998 for segment VII and 0.999 for segment VIII. The ICC for the total mean liver PDFF change was 0.998, for the PDFF change of the RLL was 0.998 and for the PDFF change of the LLL was 0.998.

\section{PDFF spatial variation at baseline and follow-up}

At baseline, the median PDFF was $7.5 \%$ in the RLL (range, $0.6-43.8 \%$ ) and $5.5 \%$ in the LLL (range, $0.8-39.9 \%$ ) (paired $t$-test: $\mathrm{P}<0.001)$. At 1 -year follow-up, the median PDFF was $9.2 \%$ in the RLL (range, 2.8-42.4\%) and 6.7\% in the LLL (range, $1.6-41.6 \%$ ) (paired $t$-test: $\mathrm{P}<0.001$ ). All results of the individual segments are shown in Table 1.

\section{Correlation between mean liver PDFF and PDFF of specific lobes and segments at baseline}

Strong correlations were observed between the mean liver PDFF and the PDFFs for the different lobes and segments $(\mathrm{P}<0.001$ for both lobes and all segments). The liver PDFF 
Table 1 Values of mean, standard deviation (SD), median and range of PDFF in \% of total liver mass, left liver lobe, right liver lobe and specific liver segments at baseline and follow-up scans

\begin{tabular}{|c|c|c|c|c|c|c|}
\hline Region & Baseline (I)/follow-up (II) & Mean (\%) & SD (\%) & Median (\%) & $\operatorname{Min}(\%)$ & $\operatorname{Max}(\%)$ \\
\hline \multirow[t]{2}{*}{ Total liver } & I & 9.8 & 9.7 & 6.4 & 0.7 & 41.7 \\
\hline & ॥ & 10.2 & 9.5 & 7.7 & 2.1 & 41.4 \\
\hline \multirow[t]{2}{*}{ Right lobe } & 1 & 10.9 & 10.1 & 7.6 & 0.6 & 43.8 \\
\hline & II & 11.1 & 9.5 & 9.2 & 2.8 & 42.4 \\
\hline \multirow[t]{2}{*}{ Left love } & I & 8.8 & 9.4 & 5.5 & 0.8 & 39.9 \\
\hline & $\|$ & 9.5 & 9.5 & 6.7 & 1.6 & 41.6 \\
\hline \multirow[t]{2}{*}{ Seg I } & 1 & 7.7 & 9.2 & 5.1 & -1.2 & 37.7 \\
\hline & ॥ & 8.3 & 9.2 & 5.8 & 1.0 & 39.7 \\
\hline \multirow[t]{2}{*}{ Seg II } & 1 & 9.4 & 9.7 & 6.2 & -0.3 & 42.1 \\
\hline & ॥ & 10.1 & 9.9 & 7.9 & 1.6 & 45.5 \\
\hline \multirow[t]{2}{*}{ Seg III } & 1 & 8.9 & 10.1 & 5.4 & 0.1 & 43.2 \\
\hline & $\|$ & 10.0 & 10.2 & 7.2 & 0.9 & 45.2 \\
\hline \multirow[t]{2}{*}{ Seg IVa } & 1 & 9.0 & 8.9 & 5.6 & 1.4 & 36.4 \\
\hline & $\|$ & 9.4 & 8.7 & 6.6 & 0.7 & 37.9 \\
\hline \multirow[t]{2}{*}{ Seg IVb } & 1 & 9.2 & 9.8 & 5.7 & 0.7 & 40.1 \\
\hline & II & 9.5 & 10.0 & 6.3 & 1.3 & 43.2 \\
\hline \multirow[t]{2}{*}{ Seg V } & 1 & 10.1 & 10.4 & 6.3 & 0.3 & 42.2 \\
\hline & II & 10.2 & 9.0 & 7.4 & 1.6 & 39.7 \\
\hline \multirow[t]{2}{*}{ Seg VI } & I & 10.9 & 9.7 & 8.2 & -0.1 & 43.0 \\
\hline & II & 10.7 & 8.4 & 9.5 & 3.0 & 38.7 \\
\hline \multirow[t]{2}{*}{ Seg VII } & 1 & 10.9 & 10.2 & 7.1 & 0.5 & 44.9 \\
\hline & II & 11.7 & 10.3 & 9.2 & 2.6 & 46.6 \\
\hline \multirow[t]{2}{*}{ Seg VIII } & 1 & 11.6 & 10.6 & 8.3 & 1.9 & 45.3 \\
\hline & ॥I & 11.7 & 10.7 & 8.8 & 2.2 & 44.7 \\
\hline
\end{tabular}

PDFF, proton density fat fraction.

of both lobes showed a close correlation with mean liver $\operatorname{PDFF}\left(\mathrm{R}_{\text {left lobe }}^{2}=0.99, \mathrm{R}_{\text {right lobe }}^{2}=0.98\right.$, respectively $)$. Highest $\mathrm{R}^{2}$ values of agreement with the mean PDFF were observed in segments $I V a\left(R^{2}=0.99\right), I V b\left(R^{2}=0.99\right)$ and VI $\left(R^{2}=0.98\right)$. The lowest $R^{2}$ values were observed in the segments I $\left(\mathrm{R}^{2}=0.96\right)$, II $\left(\mathrm{R}^{2}=0.96\right)$ and IX $\left(\mathrm{R}^{2}=0.96\right)$.

\section{Comparison of individual lobe PDFF changes}

Figure 1 shows two examples of liver PDFF maps at baseline and follow-up for a subject with increasing liver PDFF
(Figure $1 A, B$ ) and for a subject with decreasing liver PDFF (Figure 1C,D). In both subjects, the absolute PDFF changes in the RLL (white circle) were larger, compared to the absolute PDFF changes in LLL (green circle).

Figure 2 shows the correlation of the changes of PDFF in the left and the right lobe with mean liver PDFF change. The regression line of the LLL PDFF change with the mean liver PDFF change (Figure $2 A$ ) had a slope $\mathrm{m}$ of 0.93 and an intercept of $-0.23 \%$. The regression line of the RLL PDFF change with the mean liver PDFF change (Figure $2 B$ ) had a slope $\mathrm{m}$ of 1.08 and an intercept of -0.29 . 

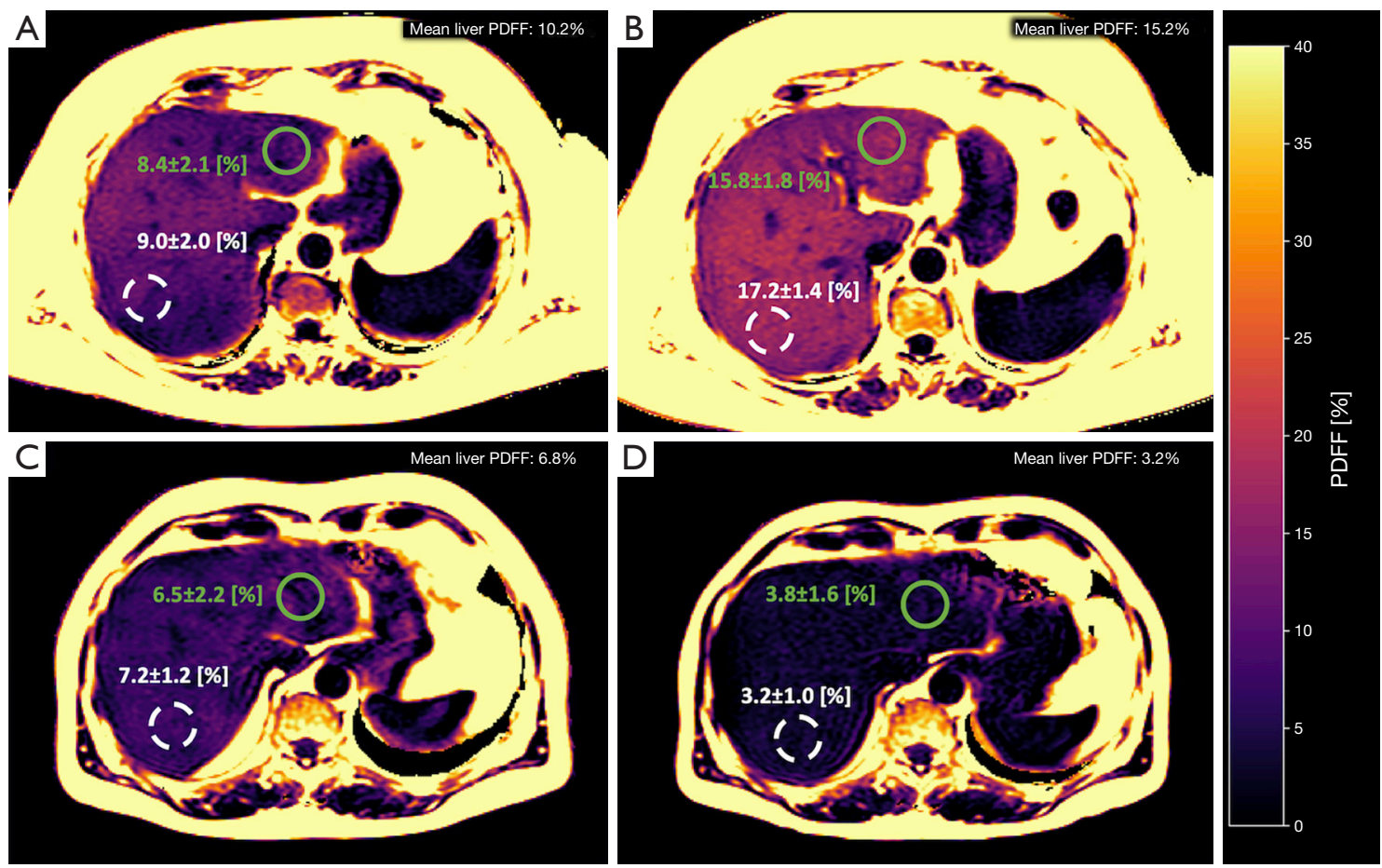

Figure 1 Liver PDFF maps at two time points (A, C at baseline and B, D after one year) for (A,B) one subject with increasing liver PDFF (weight gain of $5.9 \mathrm{~kg}$ ) and (C,D) one subject with decreasing liver PDFF (weight loss of $7.1 \mathrm{~kg}$ ). (A) shows higher PDFF in the right lobe compared to the left lobe. (B) shows the same subject after one year with strongly increasing mean liver PDFF and a larger PDFF change in the right lobe compared to the left lobe $\left(\Delta \mathrm{PDFF}_{\text {left lobe }}=7.4 \%, \Delta \mathrm{PDFF}_{\text {right lobe }}=8.2 \%\right)$. The same effect of larger changes of liver $\mathrm{PDFF}$ in the right lobe compared to the left lobe can also be detected in the subject with decreasing mean liver $\mathrm{PDFF}(\Delta \mathrm{PDFF}$ left lobe $=-2.7 \%, \Delta \mathrm{PDFF}$ right lobe $=-4.0 \%)(\mathrm{C}, \mathrm{D})$. PDFF, proton density fat fraction.
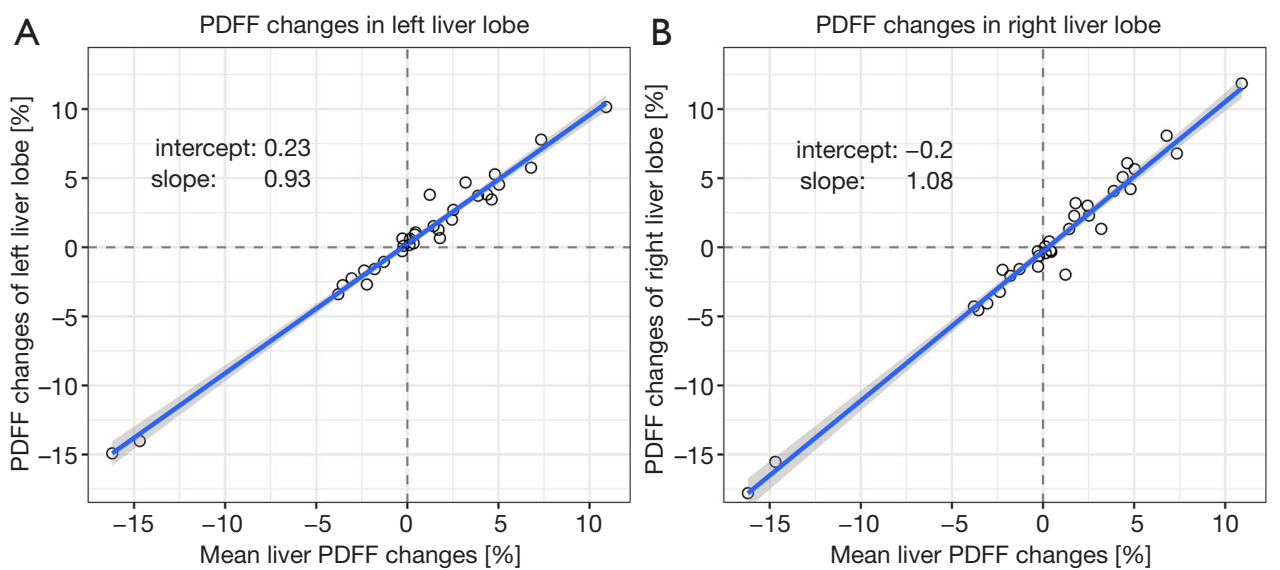

Figure 2 Changes of the liver PDFF in the LLL (A) and the RLL (B) as a function of mean liver PDFF change. The right lobe shows larger liver PDFF changes compared to the left lobe, visualized by the slopes of the regression line. PDFF, proton density fat fraction; LLL, left liver lobe; RLL, right liver lobe. 

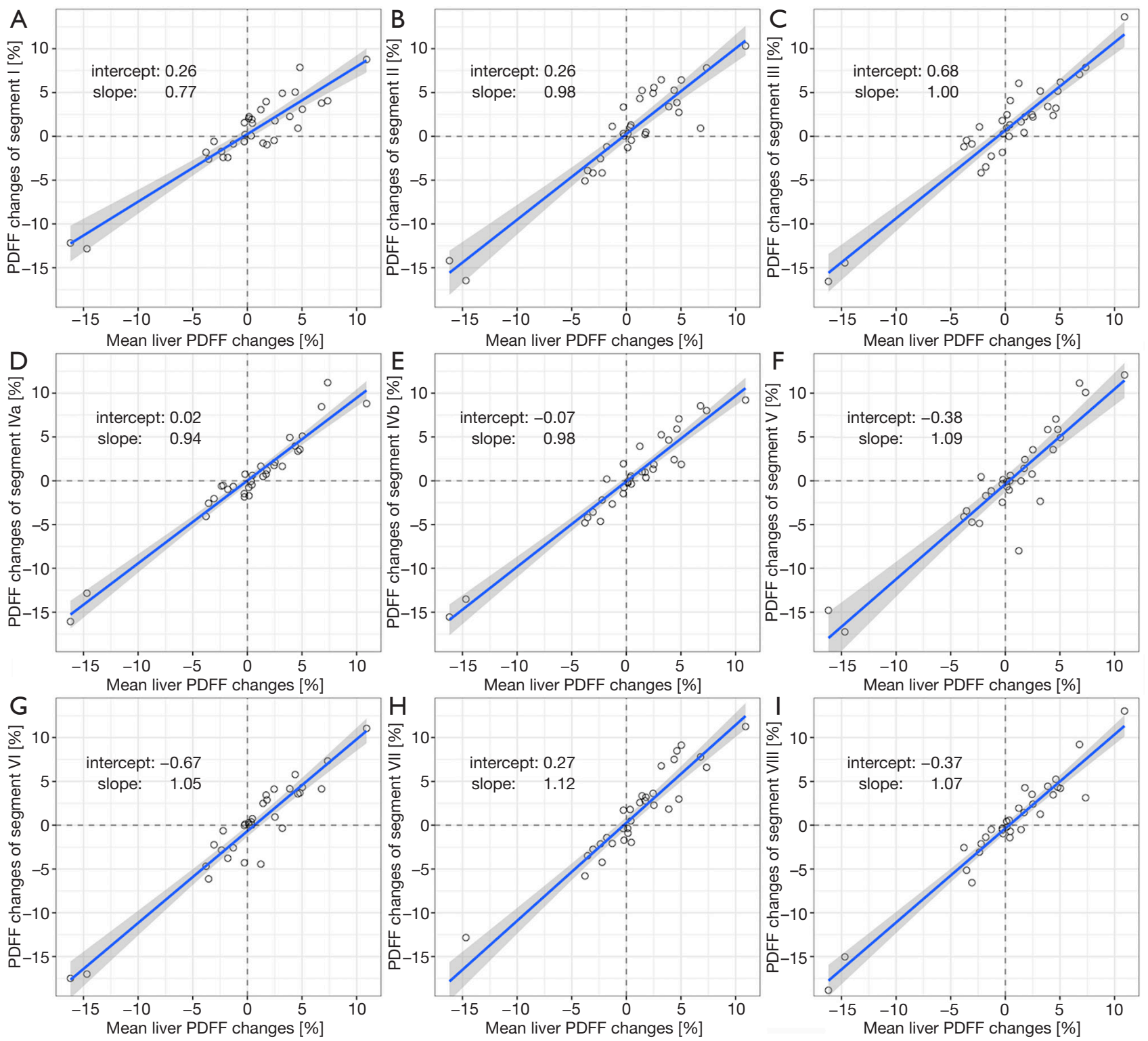

Figure 3 Changes of the liver PDFF for each by Couinaud segment (A,B,C,D,E,F,G,H,I) as a function of mean liver PDFF change. PDFF changes in segments $\mathrm{V}$ and VII show the strongest influence on the mean liver PDFF change (highest slope). PDFF changes in segment I show the weakest influence on the mean liver PDFF change (lowest slope). PDFF, proton density fat fraction.

The slope of the regression line for the correlation of RLL PDFF change versus the mean liver PDFF change was significantly higher $(\mathrm{P}<0.01)$ when compared to the slope of the agreement for the LLL PDFF change versus the mean liver PDFF change.

\section{Comparison of specific segment PDFF changes}

Figure 3 shows the correlation of PDFF changes in each
Couinaud segment compared with the mean liver PDFF changes. The highest slope of agreement between PDFF changes in each specific liver segment and mean liver PDFF changes was observed in segment VII $\left(\mathrm{m}_{\text {slope }}=1.12\right)$. The lowest slope of agreement between PDFF changes in each specific liver segment and mean liver PDFF changes was observed in segment $\mathrm{I}\left(\mathrm{m}_{\text {slope }}=0.77\right)$. The segments with higher agreement slopes were in the RLL and the segments with lower agreement were in the LLL. 


\section{Discussion}

The present work performed a longitudinal analysis of PDFF changes in subjects participating in a long-term lifestyle intervention study for weight loss to investigate regional changes in liver PDFF over a period of one year. The main finding was a larger change of PDFF in the RLL compared to the LLL for a given mean liver PDFF change. In addition, the RLL had a stronger influence on mean liver PDFF than the LLL. For a given mean liver PDFF change (including both subjects with increasing and subjects with decreasing mean liver PDFF), differences in regional changes of liver PDFF per liver segment were also observed, with highest absolute changes in segments $\mathrm{V}$ and VII-both in the RLL—and lowest absolute changes in segments I and III-both in the LLL.

Liver fat deposition assessed by biopsy is known to be spatially heterogeneous $(15,16)$ and liver PDFF has been reported as being spatially heterogeneous in previous studies $(13,14)$. To address the above spatial heterogeneity of liver PDFF, different ROI sampling methods have been reported in the literature $(25,26)$. The present method adopted the ROI sampling method introduced in (23). Inter-observer analysis in the present work showed high agreement between the two observers.

Despite some evidence for spatial heterogeneity of liver PDFF in a cross-sectional setting $(15,16)$, only a few studies have characterized how heterogeneous PDFF changes may behave in longitudinal studies. In a previous study by Fazeli Dehkordy et al. (17), it was shown that subjects with decreasing liver PDFF following bariatric surgery have a larger liver PDFF decrease in the right lobe compared to the left lobe. The results of the present study are in agreement with the study by Fazeli Dehkordy et al. Additionally, the present study shows that liver PDFF changes in the RLL contribute stronger to mean liver PDFF changes in subjects with both increasing and decreasing liver PDFF.

The exact reasons for the observed heterogeneous changes in liver PDFF remain unclear. A common explanation suggested by other studies reporting on heterogeneous liver PDFF distribution is the difference in perfusion of the respective liver segments $(13,17)$. Specifically, different studies have tried to explain the heterogeneous liver fat distribution by differences in venous blood flow in the portal vein depending if the blood's origin was mesenteric or splenic. However, the above studies show mixed results and do not offer a clear explanation for the heterogeneous PDFF distribution in the liver (27-29). Despite the lack of a definite explanation for the observed spatial heterogeneity in liver PDFF changes, the present study is in line with previous studies reporting an inhomogeneous segmental and lobar liver fat distribution in subjects with NAFLD $(13,14)$ and advocates for a high spatial resolution PDFF measurement with whole liver coverage to more accurately assess liver fat changes.

The present study has several limitations. First, the present study included participants with different levels of compliance during and after the lifestyle intervention, resulting in subjects with both increasing and decreasing mean liver PDFF over the one-year observation period. However, the key intention of the present study was to investigate regional liver PDFF changes in subjects with longitudinal mean liver PDFF changes. An additional analysis of the weight changes between the group scanned before the beginning of the intervention and after 1 year and the group scanned after the end of the intervention at two time points with an interval of one year showed no statistical differences in the extent of weight change between the two groups. Second, regional liver analysis was based on ROI drawing as suggested in previous studies $(25,26)$ and no whole liver segmentation was performed. Third, the sample size of the present study was small and further studies are required to verify the results in larger cohorts.

\section{Conclusions}

Heterogeneous long-term liver PDFF changes in the left and right lobe and the liver Couinaud segments were observed in subjects scanned longitudinally during and after a long-term lifestyle intervention study with significant weight fluctuations. Given a mean liver PDFF change, the RLL, and segments in this area, showed larger PDFF changes compared to the LLL and the respective segments. Therefore, liver PDFF measurement methods with high spatial resolution and whole liver coverage should be used to track liver fat fraction heterogeneity changes in longitudinal studies.

\section{Acknowledgments}

Funding: The present work was supported by the European Research Council (grant agreement No. 677661, ProFatMRI), the German Research Foundation (DFGSFB824/A9) and Philips Healthcare. Additional funding 
was provided by the German Center for Diabetes Research (BMBF: 01GI0925).

\section{Footnote}

Conflicts of Interest: All authors have completed the ICMJE uniform disclosure form (available at http://dx.doi. org/10.21037/qims-20-873). Dr. HH reports grants from German Ministry of Education and Research, during the conduct of the study; Dr. DCK reports grants from European Research Council, grants from Philips Healthcare, during the conduct of the study; Dr. DCK reports grants from European Research Council (No. 677661), grants from Philips Healthcare, grants from German Research Foundation (DFG-SFB824/A9), during the conduct of the study; grants from Philips Healthcare, outside the submitted work. The other authors have no conflicts of interest to declare.

Ethical Statement: The study was approved by an ethics committee and all subjects gave written informed consent before participation in the study including repeated MRI scanning.

Open Access Statement: This is an Open Access article distributed in accordance with the Creative Commons Attribution-NonCommercial-NoDerivs 4.0 International License (CC BY-NC-ND 4.0), which permits the noncommercial replication and distribution of the article with the strict proviso that no changes or edits are made and the original work is properly cited (including links to both the formal publication through the relevant DOI and the license). See: https://creativecommons.org/licenses/by-nc-nd/4.0/.

\section{References}

1. Holman N, Forouhi NG, Goyder E, Wild SH. The Association of Public Health Observatories (APHO)

Diabetes Prevalence Model: estimates of total diabetes prevalence for England, 2010-2030. Diabet Med 2011;28:575-82.

2. World-Health-Organization. Diabetes. 2018. Available online: https://www.who.int/news-room/fact-sheets/detail/ diabetes. Accessed 07.08.2020.

3. World-Health-Organization. Obesity and Overweight. 2018. Available online: https://www.who.int/news-room/ fact-sheets/detail/obesity-and-overweight. Accessed 07.8.2020.
4. Estes C, Razavi H, Loomba R, Younossi Z, Sanyal AJ. Modeling the epidemic of nonalcoholic fatty liver disease demonstrates an exponential increase in burden of disease. Hepatology 2018;67:123-33.

5. Loomba R, Sanyal AJ. The global NAFLD epidemic. Nat Rev Gastroenterol Hepatol 2013;10:686.

6. Byrne CD, Targher G. NAFLD: a multisystem disease. J Hepatol 2015;62:S47-64.

7. Tock L, Prado WL, Caranti DA, Cristofalo DM, Lederman H, Fisberg M, Siqueira KO, Stella SG, Antunes HK, Cintra IP, Tufik S, de Mello MT, Damaso AR. Nonalcoholic fatty liver disease decrease in obese adolescents after multidisciplinary therapy. Eur J Gastroenterol Hepatol 2006;18:1241-5.

8. Bacchi E, Negri C, Targher G, Faccioli N, Lanza M, Zoppini G, Zanolin E, Schena F, Bonora E, Moghetti P. Both resistance training and aerobic training reduce hepatic fat content in type 2 diabetic subjects with nonalcoholic fatty liver disease (the RAED2 Randomized Trial). Hepatology 2013;58:1287-95.

9. Adams LA, Sanderson S, Lindor KD, Angulo P. The histological course of nonalcoholic fatty liver disease: a longitudinal study of 103 patients with sequential liver biopsies. J Hepatol 2005;42:132-8.

10. Reeder SB, Cruite I, Hamilton G, Sirlin CB. Quantitative Assessment of Liver Fat with Magnetic Resonance Imaging and Spectroscopy. J Magn Reson Imaging 2011;34:729-49.

11. Middleton MS, Heba ER, Hooker CA, Bashir MR, Fowler KJ, Sandrasegaran K, Brunt EM, Kleiner DE, Doo E, Van Natta ML, Lavine JE, Neuschwander-Tetri BA, Sanyal A, Loomba R, Sirlin CB, Network NCR. Agreement Between Magnetic Resonance Imaging Proton Density Fat Fraction Measurements and Pathologist-Assigned Steatosis Grades of Liver Biopsies From Adults With Nonalcoholic Steatohepatitis. Gastroenterology 2017;153:753-61.

12. Wildman-Tobriner B, Middleton MM, Moylan CA, Rossi S, Flores O, Chang ZA, Abdelmalek MF, Sirlin CB, Bashir MR. Association Between Magnetic Resonance Imaging-Proton Density Fat Fraction and Liver Histology Features in Patients With Nonalcoholic Fatty Liver Disease or Nonalcoholic Steatohepatitis. Gastroenterology 2018;155:1428-35.e2.

13. Bonekamp S, Tang A, Mashhood A, Wolfson T, Changchien C, Middleton MS, Clark L, Gamst A, Loomba R, Sirlin CB. Spatial distribution of MRIdetermined hepatic proton density fat fraction in adults with nonalcoholic fatty liver disease. J Magn Reson Imaging 2014;39:1525-32. 
14. Sofue K, Mileto A, Dale BM, Zhong X, Bashir MR. Interexamination repeatability and spatial heterogeneity of liver iron and fat quantification using MRI-based multistep adaptive fitting algorithm. J Magn Reson Imaging 2015;42:1281-90.

15. Larson SP, Bowers SP, Palekar NA, Ward JA, Pulcini JP, Harrison SA. Histopathologic variability between the right and left lobes of the liver in morbidly obese patients undergoing Roux-en-Y bypass. Clin Gastroenterol Hepatol 2007;5:1329-32.

16. Ratziu V, Charlotte F, Heurtier A, Gombert S, Giral P, Bruckert E, Grimaldi A, Capron F, Poynard T, Group LS. Sampling variability of liver biopsy in nonalcoholic fatty liver disease. Gastroenterology 2005;128:1898-906.

17. Fazeli Dehkordy S, Fowler KJ, Mamidipalli A, Wolfson T, Hong CW, Covarrubias Y, Hooker JC, Sy EZ, Schlein AN, Cui JY, Gamst AC, Hamilton G, Reeder SB, Sirlin CB. Hepatic steatosis and reduction in steatosis following bariatric weight loss surgery differs between segments and lobes. Eur Radiol 2019;29:2474-80.

18. Schulze MB, Hoffmann K, Boeing H, Linseisen J, Rohrmann S, Mohlig M, Pfeiffer AF, Spranger J, Thamer C, Haring HU, Fritsche A, Joost HG. An accurate risk score based on anthropometric, dietary, and lifestyle factors to predict the development of type 2 diabetes. Diabetes Care 2007;30:510-5.

19. ClinicalTrials.gov. Individualized Lifestyle Intervention in Subjects with Prediabetes (PLIS). ClinicalTrials.gov. 2013. Available online: https://www.clinicaltrials.gov/ct2/show/ NCT01947595?term=PLIS. Accessed September 22, 2020 2020.

20. Ren J, Dimitrov I, Sherry AD, Malloy CR. Composition of adipose tissue and marrow fat in humans by $1 \mathrm{H}$ NMR at 7 Tesla. J Lipid Res 2008;49:2055-62.

21. Liu CY, McKenzie CA, Yu H, Brittain JH, Reeder SB.

Cite this article as: Syväri J, Junker D, Patzelt L, Kappo K, Al Sadat L, Erfanian S, Makowski MR, Hauner H, Karampinos DC. Longitudinal changes on liver proton density fat fraction differ between liver segments. Quant Imaging Med Surg 2021;11(5):1701-1709. doi: 10.21037/qims-20-873
Fat quantification with IDEAL gradient echo imaging: correction of bias from T(1) and noise. Magn Reson Med 2007;58:354-64.

22. Juza RM, Pauli EM. Clinical and surgical anatomy of the liver: a review for clinicians. Clin Anat 2014;27:764-9.

23. Tang A, Tan J, Sun M, Hamilton G, Bydder M, Wolfson T, Gamst AC, Middleton M, Brunt EM, Loomba R, Lavine JE, Schwimmer JB, Sirlin CB. Nonalcoholic fatty liver disease: MR imaging of liver proton density fat fraction to assess hepatic steatosis. Radiology 2013;267:422-31.

24. Kleinbaum DG, Kupper LL, Nizam A, Rosenberg ES. Applied regression analysis and other multivariable methods. Nelson Education; 2013.

25. Vu KN, Gilbert G, Chalut M, Chagnon M, Chartrand G, Tang A. MRI-determined liver proton density fat fraction, with MRS validation: Comparison of regions of interest sampling methods in patients with type 2 diabetes. J Magn Reson Imaging 2016;43:1090-9.

26. Hong CW, Wolfson T, Sy EZ, Schlein AN, Hooker JC, Fazeli Dehkordy S, Hamilton G, Reeder SB, Loomba R, Sirlin CB. Optimization of region-of-interest sampling strategies for hepatic MRI proton density fat fraction quantification. J Magn Reson Imaging 2018;47:988-94.

27. Groszmann RJ, Kotelanski B, Cohn JN. Hepatic lobar distribution of splenic and mesenteric blood flow in man. Gastroenterology 1971;60:1047-52.

28. Copher GH, Dick BM. Stream line phenomena in the portal vein and the selective distribution of portal blood in the liver. Arch Surg 1928;17:408-19.

29. Tsukuda T, Ito K, Koike S, Sasaki K, Shimizu A, Fujita T, Miyazaki M, Kanazawa H, Jo C, Matsunaga N. Pre- and postprandial alterations of portal venous flow: evaluation with single breath-hold three-dimensional half-Fourier fast spin-echo MR imaging and a selective inversion recovery tagging pulse. J Magn Reson Imaging 2005;22:527-33. 